\title{
Blasenkarzinom: Mitomycin kurz nach der TURBT senkt das Rezidivrisiko
}

Bei der postoperativen Behandlung des nicht invasiven Blasenkarzinoms ist das Timing entscheidend: In einer niederländischen Studie konnte das Rezidivrisiko deutlich gesenkt werden, wenn das Chemotherapeutikum unmittelbar nach der Blasenresektion eingespült wurde.

$\mathrm{B}$ is zu $82 \%$ aller Patienten mit nicht invasivem Blasenkarzinom entwickeln nach der transurethralen Tumorresektion (TURBT; ,transurethral resection of the bladder tumor") ein Rezidiv. Diepostoperative Instillation eines Chemotherapeutikums in die Harnblase soll dieses Risiko zu senken.

Einer prospektiven Multicenterstudie zufolge sollte die Instillation möglichst frühzeitig nach der Operation erfolgen: So lag die 3-Jahres-Rezidivrate bei Instillation innerhalb von 24 Stunden nach der Resektion bei 27\%; in der Vergleichsgruppe, bei der man 2 Wochen gewartet hatte, dagegen bei $36 \%$.

In der Studie war bei insgesamt 2.243 Patienten eine Mitomycin-C-Instillation (40 $\mathrm{mg}$ in $50 \mathrm{ml}$ Salzlösung) vorgenonommen worden, unmittelbar nach der Resektion bei 1.048 Patienten und 2 Wochen danach bei 1.195 .
In einer Subgruppenanalyse wurden die Patienten in 3 Kategorien eingeteilt: eine Niedrigrisikogruppe (Vorliegen eines solitären $\mathrm{pTa}$ /pT1-Tumors vom Grad 1 oder 2), eine mit intermediärem Risiko (Primärtumor Grad 3 oder ein Rezidiv) und eine Hochrisikogruppe (alle Patienten mit multiplen Tumoren).

Auch in dieser Auswertung waren die Patienten mit früher Mitomycin-Applikation im Vorteil: Bei Hochrisikopatienten betrugen die 3-Jahres-Rezidivraten 28 versus $35 \%$, bei Patienten mit intermediärem Risiko entsprechend 20 versus $32 \%$. Während die Unterschiede in diesen beiden Gruppen signifikant waren, war das bei Patienten mit geringem Risiko nicht der Fall (43 vs. $46 \%$ ). Bei diesen hatte man allerdings einen Nachbeobachtungszeitraum von 5 Jahren gewählt. Wie das Team um Judith Bosschieter betont, hatten außerdem nur die beiden Gruppen mit höherem Risiko zusätzliche Anwendungen erhalten. $\mathrm{Ne}$ benwirkungen traten unter der Mitomycin-Therapie relativ selten auf.

$\mathrm{Zu}$ erwähnen ist, dass die Studie, obwohl prospektiv angelegt, auf alten $\mathrm{Da}$ ten beruht und die Methode nicht dem Standard entspricht. „Heutzutage“, so Bosschieter und Kollegen, „würde man Hochrisikopatienten mit Bacillus Calmette-Guérin statt mit einer Chemotherapie behandeln."

In einem ergänzenden Kommentar bewerten Shandra S. Wilson und E. David Crawford den Erkenntnisgewinn aus der Studie dennoch als hoch: Die Kernfrage zum Timing der intravesikalen Chemotherapie sei eindeutig beantwortet worden [Wilson SS, Crawford ED. Eur Urol. 2017; https://doi.org/10.1016/j.eururo. 2017.09.014].

Elke Oberhofer

Bosschieter J et al. Value of an Immediate Intravesical Instillation of Mitomycin $C$ in Patients with Non-muscle-invasive Bladder Cancer: A Prospective Multicentre Randomised Study in 2243 patients. Eur Urol. 2017; https://doi. org/10.1016/j.eururo.2017.06.038

\section{Atezolizumab, eine mögliche Alternative beim Urothelkarzinom?}

\begin{abstract}
Patienten mit fortgeschritten Urothelkarzinomen, für die eine Therapie mit Cisplatin nicht infrage kommt, kann womöglich mit dem gegen PD-L1 gerichteten Antikörper Atezolizumab geholfen werden.
\end{abstract}

$\mathrm{D}$ ie Erstlinientherapie lokal fortgeschrittener oder metastasierter Urothelkarzinome stützt sich standardmäßig auf Cisplatin. Diese Behandlung kommt aber aufgrund eines verminderten Performancestatus oder von Komorbiditäten für nahezu die Hälfte der Patienten nicht infrage. Auf Alternativen wie Carboplatin sprechen die Patienten meist nur kurz an. Zudem sind diese sehr toxisch und die Überlebensraten niedrig.

Forscher der IMvigor-210-Studiengruppe gingen in einer Phase-II-Studie der Frage nach, was Atezolizumab in der Erstlinientherapie gegen fortgeschritte- ne Urothelkarzinome zu bewirken vermag. 123 Patienten wurden in die Untersuchung einbezogen, 119 erhielten eine oder mehrere Dosen des PD-L1-Antikörpers. Nach einem medianen Followup von 17,2 Monaten lag die Rate objektiven Ansprechens bei $23 \%$ (9\% komplett, $14 \%$ partiell). 19 der 27 Remissionen dauerten zum Zeitpunkt der Datenerhebung an. Ein Ansprechen war in allen Subgruppen zu beobachten, die bezüglich der PD-L1-Expression und prognostischer Faktoren wie des Metastasierungsgrads gebildet worden waren.

Das mediane progressionsfreie Überleben betrug 2,7 Monate. Das mediane
Gesamtüberleben wurde mit 15,9 Monaten angegeben.

$30 \%$ der Patienten litten unter der Therapie an Fatigue, $12 \%$ an Diarrhö, $11 \%$ an Pruritus. Es gab einen therapiebedingten Todesfall durch Sepsis, 12\% der Patienten mussten die Therapie aufgrund von Nebenwirkungen abbrechen.

Fazit: Die IMvigor-Wissenschaftler sprechen angesichts der Ansprech- und Überlebensraten sowie der Verträglichkeit von ermutigenden Ergebnissen, die den Einsatz von Atezolizumab in der Erstlinie gegen metastasierte Urothelkarzinome untermauerten. Robert Bublak

Balar AV et al. Atezolizumab as first-line treatment in cisplatin-ineligible patients with locally advanced and metastatic urothelial carcinoma: a single-arm, multicentre, phase 2 trial. Lancet. 389(10064):67-76. 